\title{
Plastic deformation induced anisotropy in metallic glasses; a molecular dynamics study
}

\author{
J. Enrique Velasco ${ }^{\mathrm{a}}$, Amadeu Concustell ${ }^{\mathrm{b}}$, Eloi Pineda ${ }^{\mathrm{a}}$, Daniel Crespo ${ }^{\mathrm{a}}$ \\ ${ }^{a}$ Dept. Física, Universitat Politècnica Catalunya - BarcelonaTech, 08860 Castelldefels, Catalonia, Spain. \\ je.velasco85@gmail.com / eloi.pineda@upc.edu / daniel.crespo@upc.edu \\ ${ }^{b}$ Department of Materials Science and Metallurgical Engineering, University of Barcelona, Spain \\ aconcustell@cptub.eu \\ *Corresponding author: Daniel Crespo Daniel.Crespo@upc.edu Tel. (34) 934134141
}

\begin{abstract}
The atomic structure of a $\mathrm{Cu}_{13} \mathrm{Ni}_{34} \mathrm{Pd}_{53}$ metallic glass was studied by molecular dynamics simulation at different temperatures along a shear deformation cycle. A simulation box of 1 million atoms was deformed in the $\mathrm{x}$ axis and then the original orthogonal shape was recovered. Directional pair distribution functions were computed in the coordinate planes and some significant directions along the shear deformation cycle. No anisotropy was found in the initial state, while post-deformation anisotropy was revealed by significant differences of the partial pair distribution functions. The analysis of atomic environments concluded that the remnant anisotropy remains constrained into the shear plane. Low temperature samples showed remnant anisotropy after the full shear sample, while close to the glass transition the anisotropy induced by the initial shear process is removed by the subsequent recovery process.
\end{abstract}

Keywords: Amorphous alloys, Aging and rejuvenation, Anisotropy, Mechanical deformation 


\section{Introduction}

Unlike crystalline alloys, metallic glasses are amorphous metallic materials with short and medium range order but lacking the crystalline structure, this generates different mechanical properties than those observed in crystalline alloys with same composition. Metallic glasses exhibit some unique mechanical properties suitable for a variety of engineering applications [1].

Metallic glasses are produced by rapid quenching from the melt, the critical cooling rate to avoid crystallization is on the order of $10^{6} \mathrm{~K} \mathrm{~s}^{-1}$ for conventional binary metallic glasses (MGs) and $10^{2} \mathrm{~K} \mathrm{~s}^{-1}$ for bulk metallic glasses (BMGs) [2]. Indeed, glasses quenched at different rates are in different isoconfigurational states, characterized by its fictive temperature $T_{f}$, and their mechanical behavior is largely dependent on $T_{f}[3,4]$. MGs, ideally isotropic, can be anisotropic due to the solidification process [5], elastic and anelastic deformation [6,7], creep [8] and plastic inhomogeneous deformation [9]. While the atomistic origin of anelastic induced anisotropy has been attributed to bond-orientation anisotropy $[8,10]$, the origin of flow induced anisotropy has not been unveiled yet. In fact, preliminary diffraction analyses suggest that flow induced anisotropy, if existent, should be very low. But, in a recent work [11], the anisotropy of metallic glasses induced during the Non-Newtonian homogenous deformation regime was explored in La, Pd and Ce based metallic glasses. In this deformation regime, at temperatures close to the glass transition $T_{g}$, the remanence of anisotropy after tensile or compressive deformations was observed for Weissenberg numbers (Wi) higher than 1-10. 
In this work we show the results of numerical simulations on a $\mathrm{Cu}_{13} \mathrm{Ni}_{34} \mathrm{Pd}_{53}$ metallic glass under shear homogeneous deformation at a very high Wi number. This composition was chosen to approach a Pd-based metallic glass, given that some of the few available experimental results have been obtained in one of these alloys [12]. It is well known that the accuracy of an atomistic simulation depends on the potential function. Many studies of MGs have adopted pairwise potentials such as the Lennard-Jones potential [13]. However, it has been reported that pairwise potentials are unable to accurately describe the properties of metals such as the Cauchy discrepancy of elastic constants, vacancy formation energies, stacking fault energies, surface structure, and relaxation behavior [14,15]. Unfortunately, no potential is available for Pd-(Ni-Cu)-P alloys, in particular for Phosphorus, but Pd, Ni and $\mathrm{Cu}$ are common elements of many metallic glasses. The simulated composition shows glass formation without structural signatures of crystalline order after the applied quenching rates and we expect it will capture the essential features of the shearing process in this family of metallic glasses. Due to the high value of Wi number, the shearing numerical experiment is expected to induce a high degree of anisotropy in the metallic glass sample, this allowing us to characterize the distinctive structural features of this anisotropy.

\section{Materials and methods}

In this study, we adopted the EAM potential to describe interatomic interactions in the simulation model. The potential consists of a simple additive potential function and an embedded function given by:

$$
U=\frac{1}{2} \sum_{i=1}^{N} \sum_{j \neq i} \phi_{i j}\left(r_{i j}\right)+\sum_{i=1}^{N} F_{i}\left(\rho_{i}\right)
$$


Where $\phi_{i j}\left(r_{i j}\right)$ is the short-range pair potential and $r_{i j}$ is the distance between atoms $i$ and $j, \rho_{i}$ is the total density and $F_{i}\left(\rho_{i}\right)$ is the embedded atom function depending on the local electronic density. The MD (Molecular dynamics) simulations were carried out using the LAMMPS (Large-scale Atomic/Molecular Massively Parallel Simulator) simulation package [16]. The potentials used are given in [17]. The sample $\mathrm{Cu}_{13} \mathrm{Ni}_{34} \mathrm{Pd}_{53}$ with a total number of 1 million atoms was prepared in multiple steps, always using the NPT ensemble. A simple cubic lattice was generated by putting the atomic species in random lattice sites. This structure was subsequently melted by heating it up to $2000 \mathrm{~K}$ and the liquid was equilibrated for 2 ns. The liquid character of the alloy was checked by visual inspection and by computation of the pair distribution function. Subsequently, the sample was quenched to $800 \mathrm{~K}, 780 \mathrm{~K}, 740 \mathrm{~K}, 720 \mathrm{~K}, 700 \mathrm{~K}, 680 \mathrm{~K}, 650 \mathrm{~K}, 300 \mathrm{~K}$ and $0 \mathrm{~K}$ at a cooling rate of $10^{13} \mathrm{~K} \mathrm{~s}^{-1}$. Then, the samples were equilibrated at these temperatures for 2 ns. The time step was 1 fs and the glass transition at this cooling rate, detected as the change of slope in the volume vs temperature evolution, was found at $715 \mathrm{~K}$.

The equilibrated samples were subjected to a shear deformation cycle on axis x. The box was deformed by keeping the lower side fixed and displacing the upper side in the $\mathrm{x}$ direction, keeping both sides parallel - shearing process -. After the maximum desired deformation was obtained, the upper side was displaced back to its original position recovering process -. The deformation cycles were performed with maximum deformations of $10 \%, 25 \%$ and $50 \%$ respective to the box length, all starting from the same initial configuration. Both shearing and recovering processes were performed at a deformation rate $\dot{\gamma}=10^{10} \mathrm{~s}^{-1}$. 
Shear strain in the samples was computed with ovito [18] following [19,20]. Changes in the atomic distributions were revealed by computing the directional pair distribution function (dPDF) computed in plane sections, defined as

$$
g(r)=\frac{1}{N_{a}} \sum_{i}^{N_{a}} \frac{1}{N_{b}} \frac{1}{2 \pi r \Delta} \sum_{j}^{N_{b}}\left(\left\langle\delta\left(\left|\vec{r}_{i j}\right|-r\right)\right\rangle\right)
$$

Here, $N_{a}$ is the number of atoms in the sample, $N_{b}$ is the number of atoms in a plane section of thickness $\Delta$, and $\vec{r}_{i j}$ is the interatomic distance. By choosing the plane sections with different spatial orientations we can distinguish the different spatial arrangements in different directions, the directional PDF computed in a plane normal to a certain s direction will be noted as s-dPDF. The thickness of the plane sections is taken as $\Delta=1.1 \AA$.

In order to determine if the observed anisotropy is spatially localized, the geometric atomic inertia tensor up to second neighbors was computed, defined as

$$
I_{k l}\left(\vec{r}_{i}\right)=\sum_{j} \frac{1}{1+\exp \left(\frac{\left|\vec{r}_{i j}\right|-2 \rho_{2}}{\varepsilon}\right)}\left(\delta_{k l}\left|\vec{r}_{i j}\right|^{2}-r_{i j k} r_{i j l}\right) \quad \text { Eq. } 3
$$

where $k$ and $l$ are the tensor components and $\vec{r}_{i j}$ is the interatomic distance whose components are $r_{i j k}$. The diagonal components $I_{k k}$ give a measure of the atomic distribution in the plane orthogonal to the $k$-axis. The logistic function is inserted as a smooth step function centered in $\rho_{2}$, the second maximum of the PDF. Note that only the atomic positions are considered, this is why we refer it as the geometric inertia tensor, as the atomic masses are neglected. 


\section{Results and discussion}

Figure 1 shows an example of the shear strain distribution of the sample deformed at $300 \mathrm{~K}$ after shearing (left) and at the end of the recovery process (right). The atomic shear strain is referred to the initial configuration of neighboring positions until a cutoff distance of $4 \AA$ [19]. Visual inspection shows a heterogeneous distribution of the shear strain but without signature of strain localization in shear bands. The inspection has been performed on multiple planes normal to different directions and for the multiple time steps through the deformation process, thus confirming that no shear bands appear during the shearing at these temperature and deformation rate conditions. This may be due to the high deformation rate, which may be preventing the formation of shear bands. In fact, shear bands were usually found by numerical simulation at lower deformation rates [20,21]. The visual inspection also indicates that the strain seems to align parallel to the shear direction during the shearing process. However, this feature is lost after recovery, which shows a featureless distribution of shear strains. This fact does not allow us to gain insight into the possible structural anisotropy in the samples.

Figure 2 shows the dPDF of planes normal to the three coordinate axes, computed along the shearing and recovery process at different temperatures. In all cases the three dPDFs coincide before the deformation processes, showing that the samples are isotropic after the quenching and successive annealing. The overall shape of the initial PDF at 700 and $720 \mathrm{~K}$ is clearly different from the one found at lower temperatures. The shoulder of the second peak and the structure of the third peak, typical of amorphous solid metals [22] vanish, and the PDF shows a liquid character. Also the extent of ordering in the structure is

clearly different. At temperatures of $680 \mathrm{~K}$ and below the oscillations in the PDF are 
plainly noticed even at $20 \AA$ distance. At 700 and $720 \mathrm{~K}$ they vanish almost completely after the 6th shell of neighbors at a distance below $15 \AA$.

The deformation protocol has a different effect at different temperatures. At low temperatures ( 0 and $300 \mathrm{~K}$ ) no particular changes are observed after shearing, but the dPDF decouple in the recovery process. The z-dPDF (computed in the xy-planes) becomes different from those computed normal to the $\mathrm{x}$ or $\mathrm{y}$ directions. Large differences are observed in the second coordination shell, but the shape of the first correlation peak is also affected, this effect on the first dPDF peak will be further discussed below. The presence of larger effects in the $\mathrm{z}-\mathrm{dPDF}$ is predictable as the shearing process is performed in the $\mathrm{xy}$ plane, and thus it is expected that the anisotropy be mostly observed in that plane. At intermediate temperatures (650 and $680 \mathrm{~K}$ ) the anisotropic effects of the shearing process are not observed any more. At these temperatures, the amorphous structure is able to accommodate to the shearing faster than the rate of distortion induced by the deformation protocol. Contrary, at $700 \mathrm{~K}$ the dPDFs decouple again but only after the shearing process, regaining isotropy after the recovery. This change in the z-dPDF indicates that the distortion of the structure in the xy plane during shearing is faster than the relaxation time needed for the liquid to regain the equilibrated structure. Finally, at $720 \mathrm{~K}$ the mobility of the system is fast enough to maintain the structural isotropy throughout the whole shearingrecovery cycle.

Figure 3 top shows the dPDF computed in planes rotated around the $\mathrm{z}$ axis at $300 \mathrm{~K}$. The z-PDF is obviously not affected by the rotation. As already shown in Figure 2, the xand y-PDF coincide before rotation, but all three dPDFs become decoupled after rotation. Rotation of $22.5^{\circ}$ and $67.5^{\circ}$ show very similar $\mathrm{x}-$ and $\mathrm{y}-\mathrm{PDF}$, and the largest decoupling is 
observed in the dPDF computed when the $\mathrm{x}$ and $\mathrm{y}$ axes are rotated $45^{\circ}$ around the $\mathrm{z}$ direction. In this case, the $\mathrm{x}-\mathrm{PDF}$ and $\mathrm{y}-\mathrm{PDF}$ are computed in the $\mathrm{x}=\mathrm{y}$ plane and $\mathrm{x}=-\mathrm{y}$ plane of the sample, respectively.

Figure 3 bottom shows a detailed view of the first dPDF peak for the same system. It can be seen that the first interatomic distance decreases in the $\mathrm{x}=\mathrm{y}$ direction and increases in the $x=-y$ direction, as expected given that in the recovery process the box is compressed in the $\mathrm{x}=\mathrm{y}$ direction and expanded in the $\mathrm{x}=-\mathrm{y}$ direction. This decoupling of the dPDFs along the two diagonals of the xy plane provokes the effect noted previously on the first peak of the z-PDF, which shows the average distribution of first neighbor distances in this plane. Figure 4 shows the dPDF of rotated axes for the sample sheared at $700 \mathrm{~K}$. As noted above, the sign of anisotropy is only observed at the end of the shearing step. At this point the position of the first maximum of the $\mathrm{x}-\mathrm{PDF}-$ computed in $\mathrm{x}=\mathrm{y}$ plane - increases, while it decreases in the $\mathrm{y}-\mathrm{PDF}-\mathrm{x}=-\mathrm{y}$ plane -; this is also coherent with the stretching of the box in the $\mathrm{x}=\mathrm{y}$ direction and the correspondent squeezing in the $\mathrm{x}=-\mathrm{y}$ direction along the shearing process.

Figure 5 shows the intensity of the diagonal elements of the geometric atomic inertia tensor of the sample deformed and restored at $300 \mathrm{~K}$ in two representative $\mathrm{xy}$ and $\mathrm{xz}$ planes. Qualitative analysis on the xy plane shows similarities between $I_{x x}$ and $I_{y y}$, whose higher values - shown as red spots - appear scattered on the sample and are often correlated. On the contrary, high values of $I_{z z}$ on the xy plane appear aligned along the $y=z$ and $\mathrm{y}=-\mathrm{z}$ directions. As for the xz plane, no correlation is found between $I_{x x}, I_{y y}$ and $I_{z z}$. This seems to indicate that anisotropy appears aligned in the directions in which the box is squeezed (during shearing) and compressed (during recovery). 
Experimental evidence [23] in metallic glasses shows two different flow regimes, inhomogeneous flow at low temperatures and homogenous flow close to the glass transition. Inhomogeneous flow is characterized by the formation of shear bands, already detected in molecular dynamics simulations [20]. As we did not find the formation of shear bands in our simulations we cannot connect the remnant anisotropy with inhomogeneous flow. Therefore, the extent of structural change under flow has to be attributed to homogeneous flow which has been extensively studied in polymer rheology $[11,24]$. The degree of structural change that can be expected is related to the dimensionless Weissenberg number, Wi:

$$
W i=\tau_{\alpha} \dot{\gamma}=\frac{\tau_{\alpha}}{\tau_{\text {shorr }}}
$$

where $\tau_{\alpha}$ is the relaxation time at the studied temperature. When $\mathrm{Wi} \ll 1$, the time for structural relaxation is comparatively short, and even under flow the liquid remains in equilibrium (there is no induced structural change); when Wi $\gg 1$, there is structural change. In this study we find four different behaviors. In the glassy state, $T<700 \mathrm{~K}$, the picture is adequate, as permanent anisotropy vanishes as temperature increases. It is noticeable that the shearing processes below the glass transition preserves the shape of the dPDF up to $20 \AA$, corresponding to several neighboring shells. That is, the atomic structure of the glassy state is able to accommodate the imposed deformation without destroying the medium range order. Unexpectedly, temporary anisotropy appears in the liquid state, $T=700 \mathrm{~K}$, vanishing again at higher temperatures. This unexpected behavior may be attributed to similar shear and relaxation times in the low temperature liquid, which does not allow the slow liquid to regain isotropy within the observed timescale. Further work is 
needed to characterize the detailed behavior of both $\tau_{\alpha}$ and $\tau_{\text {shear }}$ along the glass transition in this alloy.

Comparison to available experimental data is not easy due to several reasons. Firstly, the cooling rate of the simulation $-10^{13} \mathrm{~K} \mathrm{~s}^{-1}-$ is much higher than that of the actual $\mathrm{Pd}_{40} \mathrm{Ni}_{30} \mathrm{P}_{20} \mathrm{Cu}_{10}$ ribbons $-\sim 10^{6} \mathrm{~K} \mathrm{~s}^{-1}-$, and thus the fictive temperature of the simulated glass is higher than that of the actual ribbons. Secondly, the deformation rate in the simulation is also much higher to those attainable macroscopically. Thirdly, the effect of $\mathrm{P}$ is probably quite important in the atomic topology; $\mathrm{P}$ is a non-metallic element and, may introduce some directional bonding not present in the simulation; and last, the main effects observed in the simulation are found in the second neighbor, so the subtle changes in the first neighbor will be also reflected into subtle changes of the first peak of $S(q)$, difficult to distinguish due to the limited experimental accuracy. However, these factors may in part compensate, as the bond directionality of P may increase the remnant anisotropy while the lower quenching and deformation rates in actual ribbons may probably decrease it. The fact that remnant anisotropy is detected in simulations where the atomic potential is essentially non-directional seems to indicate that this is a disorder induced effect, not due to bond directionality, and may be an aspect present in the mechanical behavior of metallic glasses under deformation.

The interesting point of this study is the characterization of the structural details giving rise to anisotropy, which have been unveiled by the calculation of the dPDFs of different geometric planes. In real materials, the experimental PDFs are obtained from Xray and Neutron scattering techniques. In the usual experimental configuration of diffraction studies determining anisotropic structural differences, the incident beam is 
perpendicular to the loading axis of the sample in transmission geometry [6]. Therefore, the scattered interference patterns detected at a given angular sector contain the contribution of several different dPDFs. This study may help to understand the origin of the broadening of the first PDF peak and the subtle changes usually observed in these experiments.

\section{Conclusions}

Molecular dynamics simulations of shearing and recovery on a $\mathrm{Cu}_{13} \mathrm{Ni}_{34} \mathrm{Pd}_{53}$ metallic glass were performed at different temperatures. The computation of directional pair distribution functions allowed us the characterization of the structural anisotropy after the deformation process, giving a clear description of the changes underwent by the atomic environments.

The simulations were performed at high Weissenberg number at room temperature and close to the glass transition. Only the low temperature simulations, at 0 and $300 \mathrm{~K}$, showed anisotropy after recovering the original box size. Both the directional pair distribution functions and the geometric momentum of inertia computed at atomic level showed that remnant anisotropy appears aligned in the $\mathrm{x}=\mathrm{y}$ and $\mathrm{x}=-\mathrm{y}$ planes. This result is coherent with the shear applied in the xy plane.

Temporary anisotropy was observed after shear at $700 \mathrm{~K}$. However, atomic isotropy was recovered by subsequent shearing to the original box shape. The difference in the anisotropy generation at this temperature is attributed to the liquid-like structure observed at temperatures of $700 \mathrm{~K}$ and above. 
Further work will be addressed to reduce the shearing rate to study remnant anisotropy in the inhomogeneous flow regime, as well as the interplay between the structural relaxation time and the shearing rate.

\section{Acknowledgments}

Work funded by MINECO, grant FIS2014-54734-P, Generalitat de Catalunya, grant 2014SGR00581 and Barcelona Supercomputing Center and Cenits-Computaex, grants QCM-2015-1-0011, QCM-2015-2-0017 and QCM-2015-3-0038. J.E. Velasco is supported by CONACYT. 


\section{References}

[1] M. Miller, P. Liaw, eds., Bulk Metallic Glasses, Springer US, Boston, MA, 2008. doi:10.1007/978-0-387-48921-6.

[2] C. Suryanarayana, A. Inoue, Bulk metallic glasses, CRC Press, 2011.

[3] G. Kumar, P. Neibecker, Y.H. Liu, J. Schroers, Critical fictive temperature for plasticity in metallic glasses, Nat. Commun. $4 \quad$ (2013) 1536. doi:10.1038/ncomms3357.

[4] T. Egami, Formation and deformation of metallic glasses: Atomistic theory, Intermetallics. 14 (2006) 882-887. doi:10.1016/j.intermet.2006.01.004.

[5] H.R. Sinning, L. Leonardsson, R.W. Cahn, Irreversible anisotropic length changes in fe40ni40b20 and a search for reversible length changes in several metallic glasses, Int. J. Rapid Solidif. 1 (1985) 175-197.

[6] H.F. Poulsen, J.A. Wert, J. Neuefeind, V. Honkimäki, M. Daymond, Measuring strain distributions in amorphous materials, Nat. Mater. 4 (2004) 33-36. doi:10.1038/nmat1266.

[7] R.T. Ott, M.J. Kramer, M.F. Besser, D.J. Sordelet, High-energy X-ray measurements of structural anisotropy and excess free volume in a homogenously deformed Zrbased metallic glass, Acta Mater. $54 \quad$ (2006) 2463-2471. doi:10.1016/j.actamat.2006.01.026.

[8] W. Dmowski, Y. Tong, T. Iwashita, Y. Yokoyama, T. Egami, Universal mechanism of thermomechanical deformation in metallic glasses, Phys. Rev. B. 91 (2015) 60101. doi:10.1103/PhysRevB.91.060101. 
[9] H. Shakur Shahabi, S. Scudino, I. Kaban, M. Stoica, U. Rütt, U. Kühn, J. Eckert, Structural aspects of elasto-plastic deformation of a Zr-based bulk metallic glass under uniaxial compression, Acta Mater. $95 \quad$ (2015) 30-36. doi:10.1016/j.actamat.2015.05.011.

[10] W. Dmowski, T. Egami, Structural Anisotropy in Metallic Glasses Induced by Mechanical Deformation, Adv. Eng. Mater. $10 \quad$ (2008) 1003-1007. doi:10.1002/adem.200800121.

[11] Y.H. Sun, A. Concustell, M.A. Carpenter, J.C. Qiao, A.W. Rayment, A.L. Greer, Flow-induced elastic anisotropy of metallic glasses, Acta Mater. 112 (2016) 132140. doi:10.1016/j.actamat.2016.04.022.

[12] A. Concustell, S. Godard-Desmarest, M.A. Carpenter, N. Nishiyama, A.L. Greer, Induced elastic anisotropy in a bulk metallic glass, Scr. Mater. 64 (2011) 1091-1094. doi:10.1016/j.scriptamat.2011.02.028.

[13] C.C. Wang, C.H. Wong, Structural properties of ZrxCu90-xA110 metallic glasses investigated by molecular dynamics simulations, J. Alloys Compd. 510 (2012) 107113. doi:10.1016/j.jallcom.2011.07.110.

[14] M.S. Daw, M.I. Baskes, Embedded-atom method: Derivation and application to impurities, surfaces, and other defects in metals, Phys. Rev. B. 29 (1984) 64436453. doi:10.1103/PhysRevB.29.6443.

[15] F. Cleri, V. Rosato, Tight-binding potentials for transition metals and alloys, Phys. Rev. B. 48 (1993) 22-33. doi:10.1103/PhysRevB.48.22.

[16] S. Plimpton, Fast Parallel Algorithms for Short-Range Molecular Dynamics, J. 
Comput. Phys. 117 (1995) 1-19. doi:10.1006/jcph.1995.1039.

[17] X.W. Zhou, R.A. Johnson, H.N.G. Wadley, Misfit-energy-increasing dislocations in vapor-deposited CoFe/NiFe multilayers, Phys. Rev. B. 69 (2004) 144113. doi:10.1103/PhysRevB.69.144113.

[18] A. Stukowski, Visualization and analysis of atomistic simulation data with OVITOthe Open Visualization Tool, Model. Simul. Mater. Sci. Eng. 18 (2010) 15012. doi:10.1088/0965-0393/18/1/015012.

[19] M.L. Falk, J.S. Langer, Dynamics of viscoplastic deformation in amorphous solids, Phys. Rev. E. 57 (1998) 7192-7205. doi:10.1103/PhysRevE.57.7192.

[20] F. Shimizu, S. Ogata, J. Li, Theory of Shear Banding in Metallic Glasses and Molecular Dynamics Calculations, Mater. Trans. 48 (2007) 2923-2927. doi:10.2320/matertrans.MJ200769.

[21] D. Şopu, Y. Ritter, H. Gleiter, K. Albe, Deformation behavior of bulk and nanostructured metallic glasses studied via molecular dynamics simulations, Phys. Rev. B. 83 (2011) 100202. doi:10.1103/PhysRevB.83.100202.

[22] Y. Waseda, The Structure of Non-Crystalline Materials: Liquids and Amorphous Solids, Mcgraw-Hill (Tx), 1980.

[23] F. SPAEPEN, Homogeneous flow of metallic glasses: A free volume perspective, Scr. Mater. 54 (2006) 363-367. doi:10.1016/j.scriptamat.2005.09.046.

[24] J.M. Dealy, J. Wang, Melt Rheology and its Applications in the Plastics Industry, Springer Netherlands, Dordrecht, 2013. doi:10.1007/978-94-007-6395-1. 
Figure Captions

Figure 1. Shear stress distribution of the sample deformed up to $25 \%$ at $300 \mathrm{~K}$ in a xy plane, referred to the initial configuration. Left: after shearing. Right: after recovery.

Figure 2. Directional PDF in planes normal to the three coordinates at different temperatures after shearing up to $25 \%$ and subsequent recovery of the orthogonal shape. In order to help visual comparison, the PDF before and after the shear/recovery process at the same temperature are displaced vertically; initial state (down), sheared box (middle), final state (top). When the three components overlap only the z component is visible.

Figure 3. Top: directional PDF computed in the sample deformed up to $25 \%$ at $300 \mathrm{~K}$ after rotation of the box around the $\mathrm{z}$ axis. Same captions than in Figure 2. Bottom: zoom of the first correlation peak.

Figure 4 Top: directional PDF computed in the sample deformed up to at $700 \mathrm{~K}$ after rotation of the box around the $\mathrm{z}$ axis. Same captions than in Figure 2. Bottom: zoom of the first correlation peak.

Figure 5. Diagonal components of the atomic geometric inertia tensor of the sample deformed up to $25 \%$ and restored at $300 \mathrm{~K}$ on a xy (top) and xz (bottom) plane. Color scale ranges from blue (lowest value) to red (highest value). 

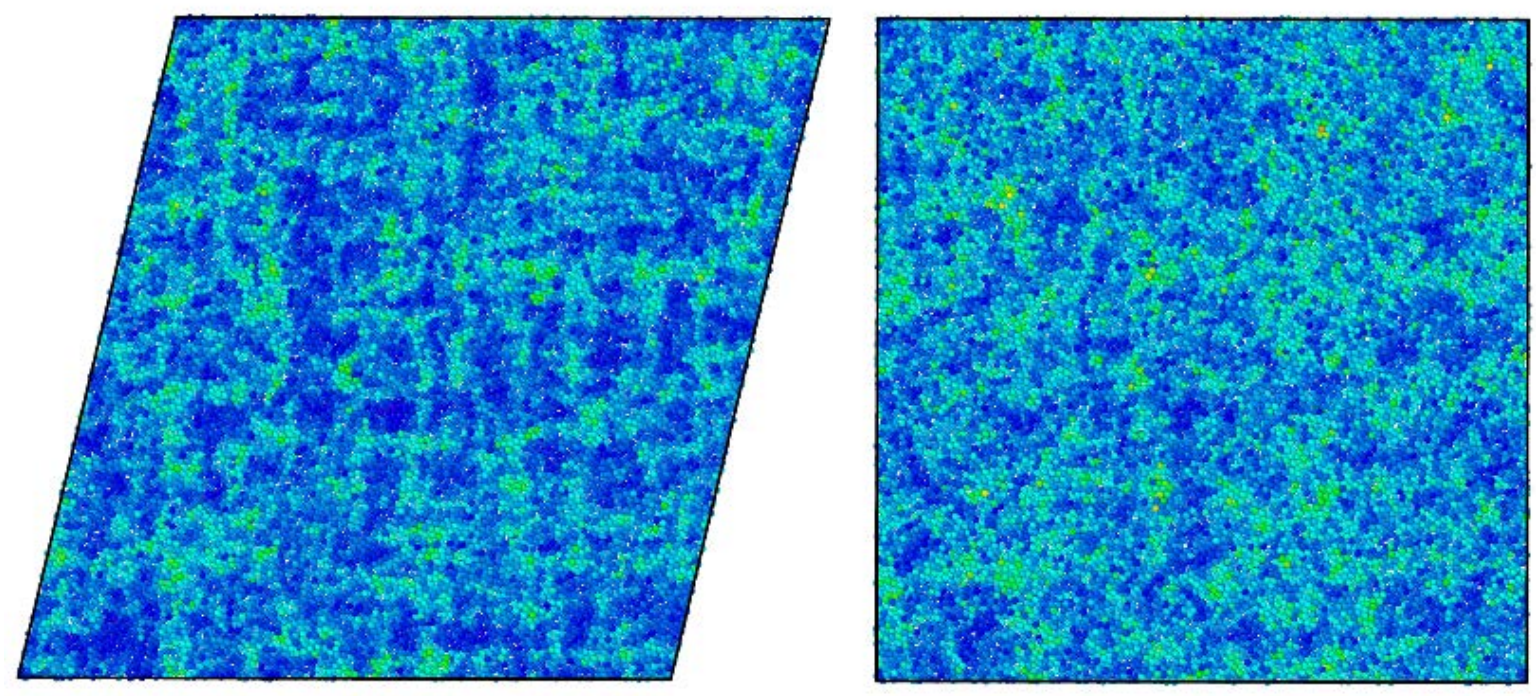

Figure 1 


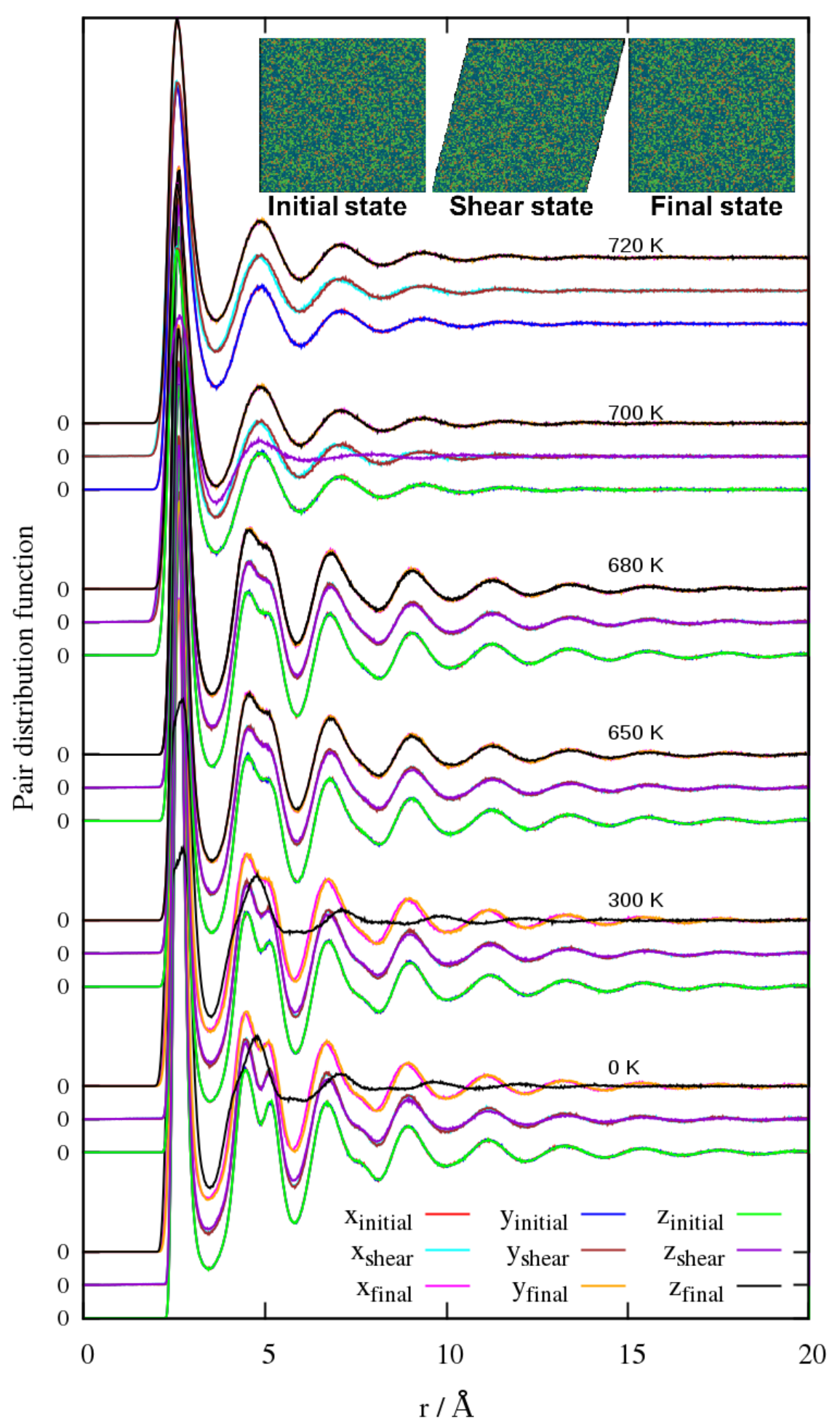

Figure 2 

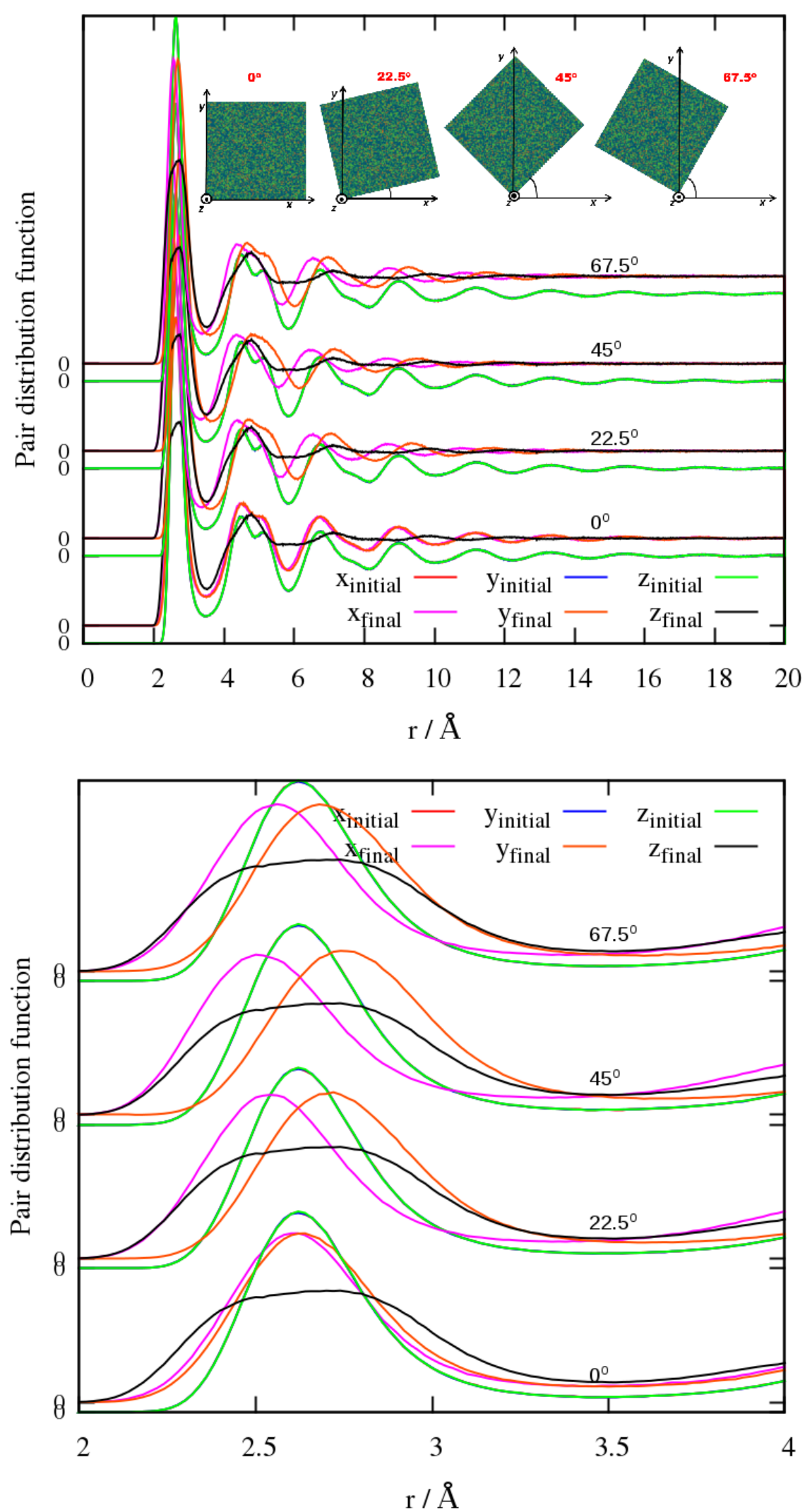

Figure 3 

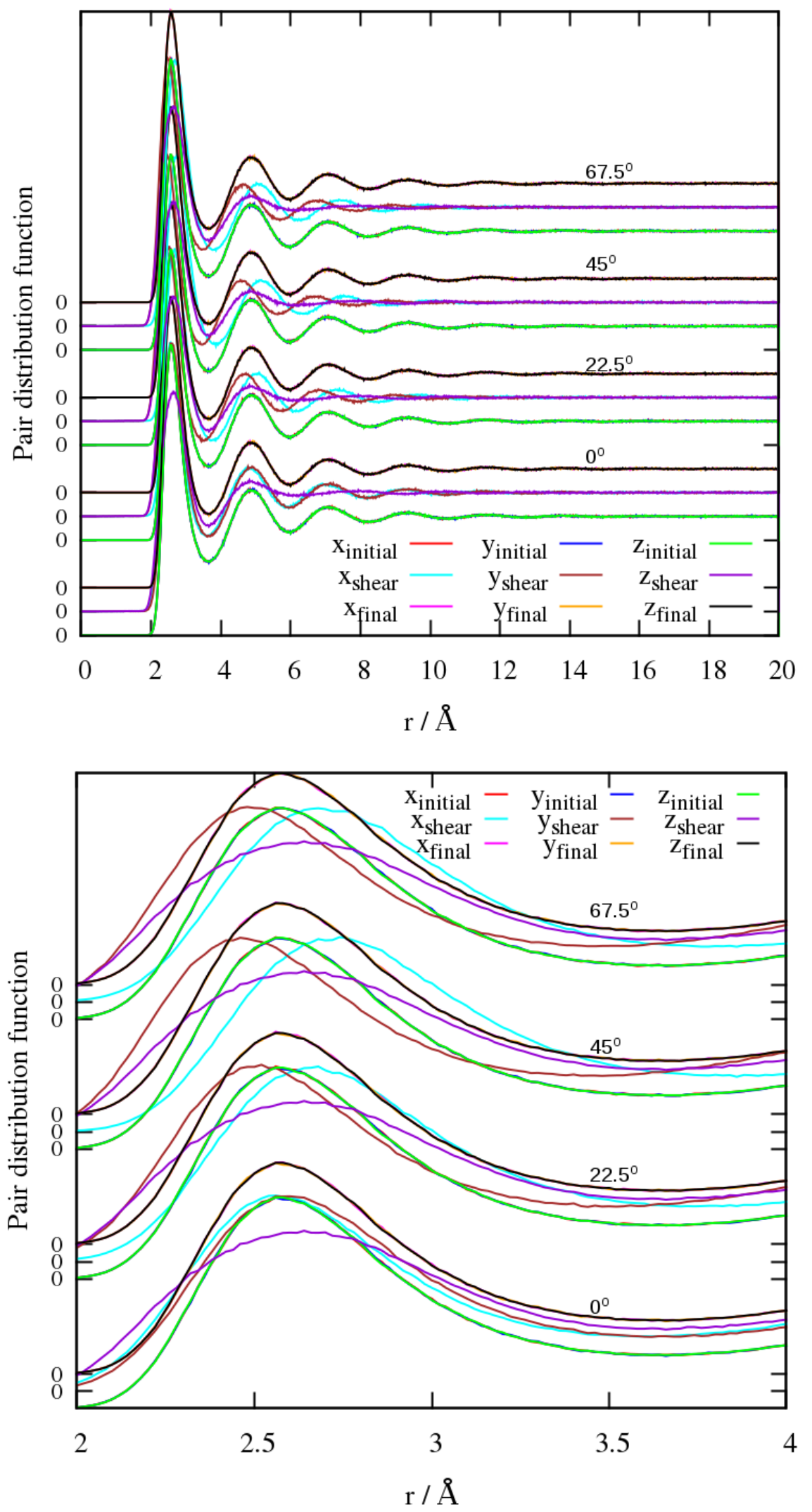

Figure 4 

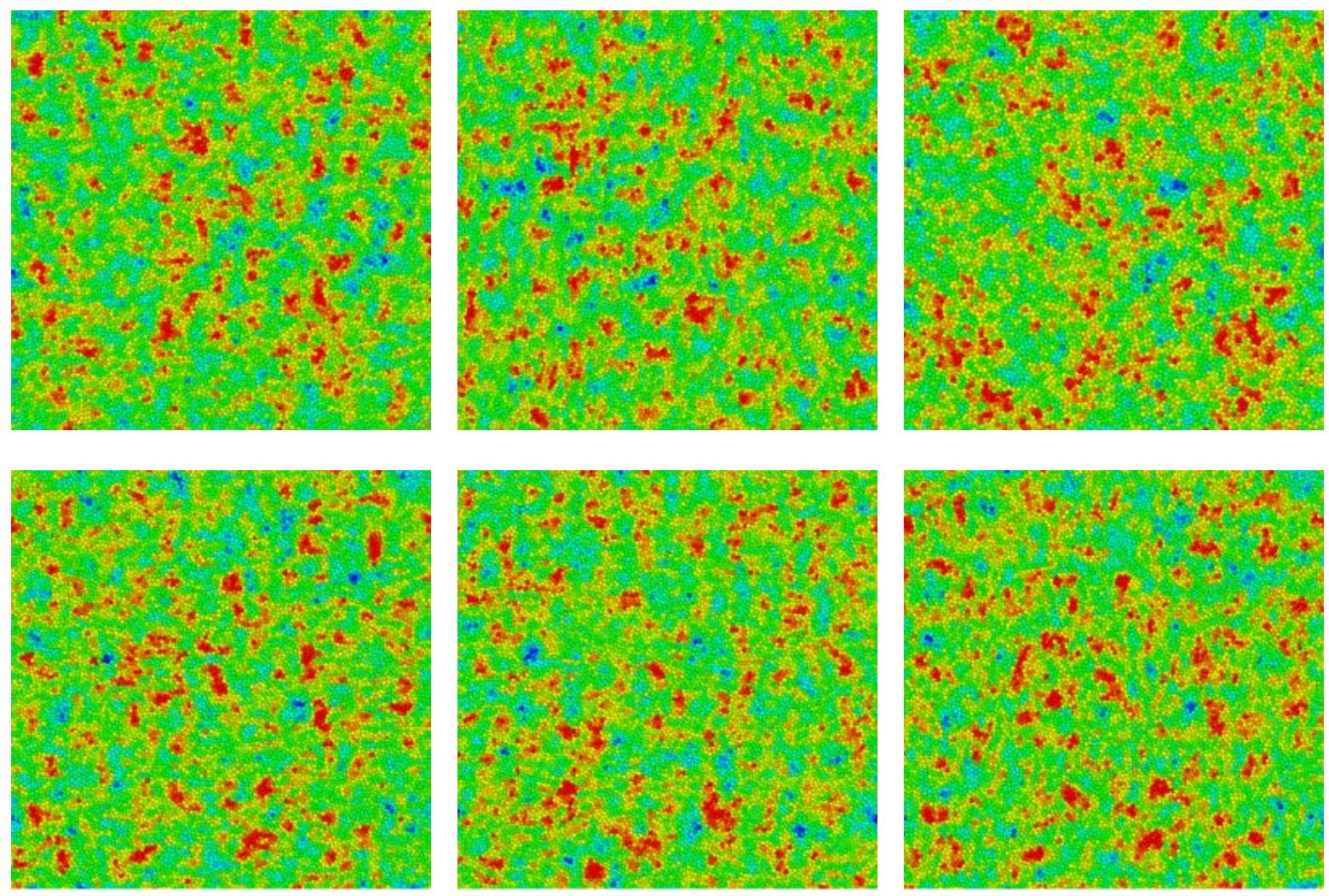

$\mathrm{I}_{\mathrm{xx}}$

Iyy

$\mathrm{I}_{\mathrm{zz}}$

Figure 5 